\title{
Phenytoin Induced Psychosis: A Case Report
}

\author{
Tenzin Tsundue ${ }^{1,2}$, Lekkala Ajith ${ }^{1,2}$, Khayati Moudgil ${ }^{1,2, *}$ \\ 'Department of Pharmacy Practice, JSS College of Pharmacy, Udhagamandalam, The Nilgiris, Tamilnadu- 643 001, INDIA. \\ 2JSS Academy of Higher Education and Research, Mysore, Karnataka, INDIA.
}

\begin{abstract}
An adverse drug reaction is a causal relationship between a drug and an event. Alcohol consumption remains a huge threat to the global health. A seizure is an event accompanied with loss of motor activity and alterations in the patients EEG. Phenytoin is one of the most commonly employed anti-seizure medications. Manifestations of chronic phenytoin use may induce phenytoin toxicity and psychosis. Here we report a case of a 45 year old male patient with probable phenytoin induced psychosis while being treated for seizures in the in-patient ward. The long term use of phenytoin may manifest psychotic symptoms in certain population. There is a definite need of more evidence in this field and proper diagnostic and management approaches.
\end{abstract}

Key words: Adverse drug reaction, Alcohol, Phenytoin, Probable, Psychosis, Seizures.

\section{Correspondence}

Dr. Khayati Moudgil ${ }^{1,2}$

${ }^{1}$ Clinical Resident, Department of Pharmacy Practice, JSS College of Pharmacy, Udhagamandalam- 643 001, The Nilgiris, Tamilnadu, INDIA. ${ }^{2}$ JSS Academy of Higher Education and Research, Mysuru, Karnataka, INDIA

Phone: +91-9489238815

Email: khayatimoudgil18@gmail.com

DOI: 10.5530/jyp.2018.10.108

\section{INTRODUCTION}

An adverse drug reaction (ADR) is an injury caused due to a drug. A causal relationship between the drug and the event signifies an ADR, which can also be due to an interaction of the drug and the events usually are unexpected with resultant toxic effects. ${ }^{1}$ Based on the severity and the outcome of an ADR, it may require a change in therapy, admission to a hospital, prolonged hospital stay that may even result in disability, coma or death. ${ }^{2}$

Alcohol use has lead to a huge burden on the global health. Alcohol withdrawal syndrome refers to a set of outcomes following reduction or abstinence from alcohol use. Such outcomes usually occur in individuals with alcohol dependency and the pathophysiology behind this generally involves a decreased responsiveness of the Gama aminobutyric acid (GABA) receptors and abnormalities in the glutamate receptors. ${ }^{3}$

Seizures can be defined as an event characterized by an altered state of consciousness that may be accompanied with loss of motor activity or abnormal motor activity with alterations in the patients electroencephalogram (EEG). ${ }^{4}$ The induction of the abnormal discharge potentiates from the neurons in the cortical or subcortical regions, that can also be triggered by minute stimuli such as fever, drug induced and biochemicalphysiological disturbances. ${ }^{5}$

Phenytoin acts via the direct action on the sodium channels receptors regulating the movement of the sodium ions resulting into net control of the action potential and neuronal firing. ${ }^{6}$ However, long-term use of anti-seizure drugs has been reported to produce psychosis in various patients. $^{7}$

Psychosis can be defined as a mental health problem leading to the inability of the patient to perceive the reality (hallucinations and delusions). ${ }^{8}$ The incidence of psychosis in India ranges up to 58.2 per thousand populations. ${ }^{9}$ The descriptions of drug-induced psychosis as complications of long-term therapy of anti-seizure drugs is still non conclusive as the number of evidential reviews are less.

\section{CASE HISTORY}

A 45 year old male patient was presented to the intensive care unit (ICU) with complaints of seizures and altered behavior. On examination his physical and vitals were normal, Central nervous system (CNS) with bilateral postural tenderness was also found to be normal, Cardio vascular sounds (CVS) S1S2 normal, Respiratory system (RS) with bilateral air entry positive and per abdomen soft. Blood pressure (BP) was measured at $110 / 70 \mathrm{mmHg}$ and Pulse Rate (PR) was 88 beats/minute. His past medical history found him to be a chronic alcoholic and with occasional seizure episodes since ten years. His medication history includes Phenytoin 100mg BD.

At the day of admission, his partial oxygen was measured 95\% in room air with fluid input and output being $1500 / 1000 \mathrm{ml}$. The patient was prescribed with IV Ringer Lactate (RL) 2 pints, Normal Saline (NS) 2 pints, Inj Diazepam $1 \mathrm{mg}$ diluted in $10 \mathrm{ml}$ of NS IV SOS, Inj Phenytoin $600 \mathrm{mg}$ in 1pint of NS infused for 1hour, Inj Ranitidine 50mg BD, IV Ondansetron 10mg SOS, Inj Vitamin $B_{12}$ 100mg IM OD, Inj Thiamine $100 \mathrm{mg}$ in 1pint Normal Saline over one hour and Inj Dexamethasone $8 \mathrm{mg}$ BD.

On the second day since admission, the BP was measured at $110 / 60 \mathrm{mmHg}$, PR-78/minute, Respiratory rate (RR)-22/minute and his partial oxygen $\left(\mathrm{SPO}_{2}\right)$ was $95 \%$ in room air with fluid input and output chart being 2400/1600 ml. He was prescribed with Inj Ranitidine 50mg BD, Inj Vitamin B12 100mg IM OD, Inj Dexamethasone 8mg BD, Tab Phenytoin 100mg, Tab Calcium 300mg OD, Tab B-Complex BD, Tab Diazepam 5mg 3-3-4, Inj Phenergan 12.5mg IM BD and Tab Haloperidol $1.5 \mathrm{mg}$ BD.

The next day, the patient was found to be restless and non-cooperative. Diazepam was withheld with continuation of the other therapies. On the fourth day, the patient presented with complaints of sleep disturbances and progressive muscular atrophy (PMT). BP was measured at 120/80mmHg, PR-80/min, RR-20/Min, Fasting blood Sugar (FBS)- 
110mg\%, Post Prandial Blood Sugar (PPBS)-120mg \% and his partial oxygen was $95 \%$ in room air with fluid input and output being 1250/900 $\mathrm{ml}$. The patient was prescribed with IVF NS one pint, $10 \%$ DNS 1 pint, Inj Thiamine $100 \mathrm{mg}$ in $100 \mathrm{ml}$ of normal saline, Inj Ranitidine 50mg BD, Tab Diazepam 5mg 3-3-4, Inj Phenergan 12.5mg IM BD, Tab Haloperidol 1.5mg BD and Tab B-Complex 2-2-2.

On the fifth day, the patient had complaints of bed-wetting and was not willing for catheterization. The patient was transferred to a tertiary care hospital for further treatment.

\section{DISCUSSION}

Alcohol use remains the fourth leading cause of preventable deaths in the World. The global scenario dictated excessive alcohol use linked with approximately 3.3 million deaths in the year of 2015. Moreover, alcohol consumption has been attributed to $5.1 \%$ of the global disease burden, which can be measured as in disability adjusted life years (DALYs). ${ }^{10}$ The incidence of death and disability occur relatively in the early ages in life. Similarly, an approximate of $25 \%$ of the total deaths in people from the age group 20 - 39 years were attributable to alcohol ${ }^{11}$ Moreover, alcoholism may associate with Wernicke encephalopathy, Korsakoff syndrome and hepatic encephalopathy. ${ }^{12}$ Alcohol affects virtually every organ system in the body and several neurotransmitter systems in the brain, including opiates, GABA, glutamate, serotonin and dopamine. However, chronic alcohol consumption leads to the blockade of glutamate receptors with resultant follow of complex biological pathway that ultimately leads to the over expression of glutamate receptors. Therefore, on withdrawal, the central nervous system experiences increased excitability. ${ }^{13}$ Alcohol dependence is regarded as the final phase of habitual alcohol consumption. Benzodiazepines are usually the first line choice of drug for the symptomatic reliefs, which may include an add on anticonvulsant therapy with or without supportive care depending upon the biological assay of the patients blood. ${ }^{14}$

Consumption of alcohol promotes seizure threshold, cessation of alcohol decreases the seizure threshold. The increase in seizure threshold may be mediated through the calcium and chloride flux through the ion-gated glutamate, N-methyl-D-aspartate (NMDA) and Gamma-Aminobutyric Acid (GABA) receptors. Daily alcohol intake has been reportedly linked with hyponatremia and hypomagnesaemia. ${ }^{15}$ Approach generally includes one or all of the following. Intravenous (IV) line of 5\% dextrose and saline with Inj thiamine, dextrose and Inj naloxone. Lorazepam is usually first line choice for symptomatic seizures. ${ }^{16}$

A seizure is a sudden and uncontrolled electrical disturbance induced due to imbalance in the excitatory and inhibitory forces with excessive excitation or loss of inhibition within the cortical neurons. ${ }^{17}$ Recent evidence on epileptic medications witness patients with drug induced neurological disorders. Commonly used anti-seizure medications were reported to cause antiepileptic drug induced psychotic disorder (AIPD). ${ }^{18}$ A need that arises, now is the need of more prominent evidence and regular Psychosis screening as well. However, the confirmation of the phenytoin-induced psychosis is a debatable issue and the most commonly used ways for confirmation include drug de-challenge and re-challenge.

Phenytoin is one of the most commonly used anti-seizure drug and also been reported with antiarrhythmic and muscle relaxant properties as well. ${ }^{19}$ In the year 2015, the American National Poison Data system reported 1601 patients bearing single exposures to phenytoin and moreover, 543 of them were reported with adverse reactions. ${ }^{20}$ Other indications of phenytoin include as first-line or an adjunctive therapy for partial and generalized seizures, status epilepticus, Lennox-Gastaut syndrome and childhood epileptic syndromes. Phenytoin has a very partial gastro intestinal (GI) absorption. Moreover, phenytoin, when administered intravenously at a rate higher than $50 \mathrm{mg} / \mathrm{min}$ may lead to cardiac toxicity and hypotension. The volume of distribution reported for phenytoin is $0.6 \mathrm{~L} / \mathrm{kg}$ and is also reported with $90 \%$ extensive binding to plasma proteins. ${ }^{21}$

Psychosis is a socio-economical problem associated with situations that are not real and imaginary, which affects one's personal life, social status and the people around them. Psychosis can be classified into two sub categories. Episodic psychosis of epilepsy and non-episodic psychosis of epilepsy. Episodic psychosis of epilepsy is correlated to the seizure episodes or events and vice versa. ${ }^{22}$ Patients with chronic epilepsy may or may not display psychosis related to treatment with Phenytoin. The pathophysiology rendering the relation between psychosis and phenytoin have always hypothesized with Vitamin B12 deficiency and folic acid deficiency. ${ }^{23}$ However, there are still reports of phenytoin induced psychosis non attributable to the above. Moreover one hypotheses also remains with the susceptibility linked to the neuro-anatomical anomalies that may poteintiate an drug induced psychosis. ${ }^{24}$ And a pathophysiology concerned with vitamin B deficiency in phenytoin use. ${ }^{25}$ This case signifies the long-term use of phenytoin and resultant psychosis. The psychotic symptoms were controlled upon de-challenge of the suspected drug. On the basis of Naranjo Causalty assessment, the reaction was found to be a probable reaction. The data for re-challenge was not attained because of patient transfer to a tertiary care hospital.

\section{CONCLUSION}

The following case signifies the occurrence of unexpected ADRs that might follow phenytoin use. More updated evidence and directive management approaches are a required goal in such circumstances.

\section{ACKNOWLEDGEMENT}

The authors acknowledge the support and cooperation provided by the Government Headquarters Hospital, Ooty and its esteemed doctors for their support. I would like to thank and acknowledge my HOD Dr. S. Ponnusankar for his guidance, encouragement and his immense support.

\section{CONFLICT OF INTEREST}

The authors declare no conflict of interest.

\section{ABBREVIATIONS}

ADR: Adverse drug reaction; AIPD: Antiepileptic drug Induced Psychotic Disorder; CVS: Cardio vascular sounds; CNS: Central nervous system; DALYs: Disability adjusted life years; EEG: Electroencephalogram; FBS: Fasting blood Sugar; GABA: Gama aminobutyric acid; GI: Gastro intestinal; ICU: Intensive care unit; IV: Intravenous; NMDA: N-methylD-aspartate; NS: Normal Saline; PPBS: Post Prandial Blood Sugar. PMT: Progressive muscular atrophy; RS: Respiratory system; RL: Ringer Lactate.

\section{REFERENCES}

1. Morimoto $T$, Gandhi $T$, Seger A, Hsieh $T$, Bates D. Adverse drug events and medication errors: detection and classification methods. Qual Saf Health Care. 2004;13(4):306-14.

2. Zaki SA. Adverse drug reaction and causality assessment scales. Lung India : Official Organ of Indian Chest Society. 2011;28(2):152-3.

3. Becker HC. Effects of Alcohol Dependence and Withdrawal on Stress Responsiveness and Alcohol Consumption. Alcohol Research : Current Reviews. 2012;34(4):448-58.

4. Shasha L, Weihua Y, Yang L. The causes of new-onset epilepsy and seizures in the elderly. Neuropsychiatr Dis Treat. 2016;12:1425-34.

5. Tunnicliff G. Basis of the antiseizure action of phenytoin. Gen Pharmacol 1996;27(7):1091-7. 
6. Siddhartha N. Orrin D. Psychotropic Effects of Antiepileptic Drugs. Epilepsy Curr. 2005;5(5):176-181.

7. UK. Psychosis and Schizophrenia in Adults: NICE Clinical Guidelines. 2014;178(2).

8. Venkataswamy MR. Chandrashekar. Prevalence of Mental and Behavioural Disorders in India: A Meta-Analysis. Indian J Psychiatry. 1998;40(2):149-57.

9. Suresh B, Ravindra S. Indian Psychiatric epidemiological studies: Learning from the past. Indian J Psychiatry. 2010;52(1):S95-S103.

10. Tanmay N, Arnab G. Cardiovascular disease risk factors in Asian Indian population: A systematic review. J Cardiovasc Dis Res. 2013;4(4):222-8.

11. Robert $O$. Alcohol and the Nervous System. Aminoff's Neurology and General Medicine. 2014;(5):713-24.

12. Rao $P$, Richard $L$, Eric A, Youssef $S$. Targeting glutamate uptake to treat alcohol use disorders. Front Neurosci. 2015;9:144.

13. Ankur S, Mona C, Mina C. Alcohol Withdrawal Syndrome: Benzodiazepines and Beyond. J Clin Diagn Res. 2015;9(9):VE01-VE07.

14. Hillbom M, Pieninkeroinen, Leone M. Seizures in alcohol-dependent patients: epidemiology, pathophysiology and management. CNS Drugs. 2003;17(14):1013-30.

15. Eugen T, Julia H, Markus L, Francesco B. Pharmacotherapy for Status Epilepticus. Drugs. 2015;75(13):1499-521.

16. Marvin M. Overview of Drugs Used For Epilepsy and Seizures. Etiology,
Diagnosis and Treatment. PT. 2010;35(7):392-415.

17. Ziyi C, Ana L, Terence J. Psychotic disorders induced by antiepileptic drugs in people with epilepsy. Brain. 2016;139(10):2668-78.

18. Lopamudra C, Arpita L, Tamonash C, Suparna C. Chronic phenytoin therapyinduced vecuronium resistance. Indian J Pharmacol. 2011;43(2):214-5.

19. Mowry J, Spyker D, Brooks D. Annual Report of the American Association of Poison Control Centers National Poison Data System (NPDS): $33^{\text {rd }}$ Annual Report. Clinical Toxicology. 2015:54(10):924-1109.

20. Metabolism D. DILANTIN 125mg / 5mI ORAL SUSPENSION. 2016;1-12.

21. Weisholtz D, Dworetzky B. Journal of Neurological Disorders and Stroke Epilepsy and Psychosis. J Neurol Disord Stroke. 2014;2(3):1-8.

22. Linnebank M, Moskau S, Semmler A, Widman G, Wagner B, Weller M, et al. Antiepileptic drugs interact with folate and vitamin B12 serum levels. Annals of Neurology. 2011;69(2):352-9

23. Manish B, Pravin R, Sumit K. Psychosis as Harbinger of Phenytoin Toxicity. Toxicol Int. 2015;22(1):160-1.

24. Valle M, Cortés C, Santana A, Barber M, Figueras T, García H. Epileptic status refractory to conventional treatment caused by vitamin $B 6$ deficiency. Journal of Perinatology. 2009;29(3):252-3.

Article History: Submission Date : 15-05-2018; Revised Date : 08-06-2018; Acceptance Date : 16-08-2018.

Cite this article: Tsundue T, Ajith L, Moudgil K. Phenytoin Induced Psychosis: A Case Report. J Young Pharm. 2018;10(4):491-3. 